Cahiers de recherches médiévales

\title{
La « Médiévalisation » de la littérature belge de langue française, un archétype flamand
}

\section{Anna Soncini}

\section{(2) OpenEdition}

Édition électronique

URL : https://journals.openedition.org/crm/1813

DOI : 10.4000/crm.1813

ISSN : 1955-2424

Éditeur

Honoré Champion

Édition imprimée

Date de publication : 15 décembre 2004

Pagination : 151-164

ISSN : 1272-9752

\section{Référence électronique}

Anna Soncini, «La « Médiévalisation » de la littérature belge de langue française, un archétype

flamand ", Cahiers de recherches médiévales [En ligne], 11 | 2004, mis en ligne le 10 octobre 2007, consulté le 15 décembre 2022. URL : http://journals.openedition.org/crm/1813 ; DOI : https://doi.org/ $10.4000 / \mathrm{crm} .1813$

Ce document a été généré automatiquement le 15 décembre 2022.

Tous droits réservés 


\title{
La « Médiévalisation » de la littérature belge de langue française, un archétype flamand
}

\author{
Anna Soncini
}

1 Le Moyen Âge dans la littérature du XIX ${ }^{e}$ siècle, que ce soit en France, en Italie, ou en Allemagne, a toujours été une « reprise » : parfois considéré comme une continuation de la vague romantique, parfois comme une dissociation de celle-ci, parfois encore comme relevant fondamentalement d'une simple érudition, il s'agissait toujours d'exploiter un monde, une culture du passé. «Le XIX siècle redécouvre le Moyen Âge. Il l'invente, le transfigure et le "répand" »'. Cependant, quand on aborde la littérature belge, le discours se modifie car on touche à l'archétype même de cette littérature, qui trouve en cela son unité profonde, quoique quelque peu estompée aujourd'hui. Le bref parcours que je propose en ces pages vise certains écrivains parmi les plus renommés de cette littérature (choisis à titre d'exemple), pour y déceler les éléments m'aidant à définir l'importance du Moyen Âge dans leurs œuvres, le rôle archétypal que la culture de cette période revêt en Belgique et sa persistance. Je propose ainsi de suivre trois étapes chronologiquement successives l'une à l'autre : la première vise un Moyen Âge repris sans doute involontairement; la deuxième, noyau de mon discours, vise un Moyen Âge dont la conscience fait une large place à la volonté d'une recherche identitaire ; la troisième retrouve une position plus proche de celle de la France de la fin du XIX'e siècle, d'un Moyen Âge stéréotypé constitutif de la conscience d'un manque.

Un Moyen Âge non temporel

En Belgique, la culture est composite ; liée à deux langues, le flamand et le français, elle paraît se fonder sur des éléments non hiérarchisés, mais simplement juxtaposés les uns avec les autres. Autrefois, mais surtout au $\mathrm{XV}^{\mathrm{e}}$ siècle, chacune des deux langues empiétait profondément le territoire de l'autre et les deux littératures connaissaient le même éclat: "des deux côtés de la frontière linguistique, les Rhétoriqueurs, ici en français, là en thiois, firent preuve de la même fécondité et jouirent d'une vogue égale $»^{2}$. La littérature suit le même cours : toute la vie est pénétrée, en Flandre, des 
usages de la vie française. Ce moment identitaire flamand, fort conscient de l'importance de la culture française, n'est pas vite oublié. Le Moyen Âge a toujours gardé son attrait, surtout dans le folklore et dans la tradition orale, et non seulement dans la période qui a suivi, celle qui a conduit au lyrisme moderne. La stagnation des siècles passés, liée aussi à une situation politique non favorable au discours unitaire, fit en sorte que ce sentiment ne réapparut avec force que seulement lors de la naissance de l'état belge (1830). Tout au long du XIX siècle, la culture d'inspiration française montre son hégémonie en Belgique et offre à tous les écrivains, y compris à ceux d'origine non francophone, une langue littéraire ; un matériau mythique, un répertoire de l'imaginaire qui plongeaient leurs racines dans le lointain Moyen Âge de la tradition flamande: tradition fondée sur une culture populaire devenue statique, ayant été bouleversée par les événements historiques. La passion pour le Moyen Âge, que le romantisme déclare au même moment où naît l'État belge et la tentative de ce dernier de se donner une culture nationale, permet alors à la langue littéraire française d'englober le matériau d'origine flamande pour des raisons esthétiques, et non pour des raisons de localisation. L'affirmation habituelle d'une littérature belge qui se régionalise par sa langue, sa syntaxe incorrecte, sa définition territoriale, n'est que la surface d'une évolution complexe bloquée pendant des siècles par les événements historiques. C'est suite à cela que prend forme un des éléments caractérisant la littérature belge de langue française : sa « médiévalisation ».

Comme par un effet d'optique, une distorsion se produit qui superpose le Moyen Âge au matériau flamand pris en dehors ou au-delà du Moyen Âge même. Il suffit de regarder le roman fondateur de la culture belge de langue française: «La légende de Thyl Ulenspiegel $»^{3}$ de Charles De Coster. L'histoire se déroule à l'époque de Charles V, héros à facettes multiples, à un moment où l'Europe connaît la pleine maturité de la Renaissance. Cependant, le lecteur, au-delà des références spatio-temporelles, n'a pas la sensation de se trouver dans un contexte Renaissance: l'atmosphère y est encore entièrement médiévale ${ }^{4}$, ainsi qu'elle a été bien perçue par le metteur en scène du film russe $^{5}$. On ne trouve rien du magnifique essor qui fut celui du règne de Charles $\mathrm{V}$, de la métropole anversoise, rien de cette beauté du monde qui a rempli les tableaux de l'époque. Certes, on peut dire (et c'est souvent le cas) que ce texte renvoie plutôt au truculent du XVII ${ }^{\mathrm{e}}$ siècle, mais le texte semble nous inviter à regarder plus en arrière. Il nous offre l'exotisme de la chanson de geste et de la géographie fantaisiste qui se déploie à l'horizon de l'imaginaire, avec des arbres et des animaux fabuleux et une humanité monstrueuse et saisissante. Les rappels sont parfois liés à l'imaginaire moyenâgeux stéréotypé : la postulation diabolique des sorciers ${ }^{6}$, la croyance aux sorts et aux augures; la description du monde flamand visant un Moyen Âge sombre et pervers ${ }^{7}$. Souvent, toutefois, les rappels sont plus précis : les femmes-fleurs du Roman d'Alexandre $e^{8}$, surgissant au printemps sous les arbres, s'estompent en fond de toile face aux filles-fleurs de De Coster'. Les rappels peuvent aussi remonter aux souvenirs les fabliaux ${ }^{10}$. Cet esprit, où le style bas côtoie le style courtois, où l'obscène et le scabreux sont cachés par le goût marqué de la farce, fait revivre l'esprit comique et satirique d'antan. Le Moyen Âge qu'il met en scène (lors de la révolte des Pays-Bas) n'est pas un «revival» d'image, mais la représentation d'un monde intérieur qui dit la force de l'imaginaire flamand depuis toujours.

Le Moyen Âge récupéré

4 La coïncidence entre Moyen Âge et imaginaire flamand produit à la fin du XIX ${ }^{e}$ siècle une osmose : le Moyen Âge étant à la mode, le succès des belges, héritiers de De Coster, 
ne fait que grandir. On ne leur reconnaît pas, cependant, une identité historique ; leur âme du nord vient du monde anglo-saxon: Maeterlinck est le nouveau Shakespeare. Mais là justement réside le problème : le renvoi au monde anglo-saxon est un voile que la critique pose sur le monde belge, et laisse en arrière-plan l'admiration que Maeterlinck avait pour Ruysbroeck « l'admirable ». La force du Moyen Âge qui explosait à travers ce mystique le captivait : « il ignorait le grec et le latin. Il était seul et pauvre. Et cependant, au fond de cette obscure forêt brabançonne, son âme, ignorante et simple, reçoit, sans qu'elle le sache, les aveuglants reflets de tous les sommets solitaires et mystérieux de la pensée humaine $»^{11}$. Certes, on peut affirmer qu'il y a là une vision narcissique ou un espoir de pouvoir faire de même, car « Ruysbroeck a réveillé, après un repos de plusieurs siècles, non pas ce genre de pensée ${ }^{12}$, car ce genre de pensée ne sommeille jamais, mais ce genre de parole qui s'était endormi sur les montagnes où Plotin ébloui l'avait abandonné en se mettant les mains sur les yeux, comme devant un immense incendie $»^{13}$. Plotin, ce «mystique analytique» (les mystiques étant « les plus purs diamants du prodigieux trésor de l'humanité $»^{14}$ ) et la compréhension des phénomènes les plus insolites de l'âme humaine, voilà les bases sur lesquelles le Moyen Âge devient la période fondamentale à laquelle faire référence: «Il y fait extraordinairement froid ; il y fait extraordinairement sombre, et cependant, vous n'y trouverez autre chose que des flammes et de la lumière $»^{15}$. Car les idées artificielles seules s'élèvent « en de réelles ténèbres et ne prospèrent qu'aux époques littéraires et dans la mauvaise fois de siècles trop conscients, lorsque la pensée de l'écrivain demeure en deçà de ce qu'il exprime $»^{16}$. Cependant Maeterlinck n'a pas le courage d'aller jusqu'au bout de son raisonnement et entre dans le jeu symboliste des correspondances, qui - paradoxalement - n'est pas le sien. Il est convaincu que son époque a encore des mystiques, dont Mallarmé ${ }^{17}$. La rigueur spirituelle que le poète français affiche, la lutte de l'esprit contre la matière, sa préoccupation métaphysique d'abolir le hasard pour parvenir au véritable infini, lui font affirmer une correspondance d'esprit qui n'en est pas une. Mallarmé jouait contre l'impuissance de l'homme, Maeterlinck était fasciné par la force de l'intelligence humaine, par la pensée qui ne connaît pas d'obstacle temporel, mais il était en même temps écrasé par le pouvoir de la parole sacrée qui sait percer l'âme à travers les siècles. Ainsi, même s'il entretient des rapports avec le monde littéraire français, ce n'est que dans le Moyen Âge flamand que le poète belge puise sa force. Ruysbroeck lui apporte toute "l'existence $\mathrm{du}$ beau Moyen Âge noir, au moment où Dieu a été le plus surnaturellement aimé dans l'absence de tout ce qui n'était pas Dieu seul. Cela a été réellement alors l'unique été des cœurs, et à nous qui sommes en hiver, hélas !, et peutêtre plus loin, et qui souffrons même à entrer en l'automne de l'Imitation, comme si nous ouvrions une serre au sortir d'une cave, ces ardeurs semblent à présent de fiévreuses ténèbres où notre pauvre âme périrait comme une plante du pôle au soleil. C'est tristement ainsi ; et notre amour malade est devenu symbolique, au lieu que le leur était absolu, car les meilleurs d'entre nous ne peuvent plus aimer Dieu qu'en le voyant en leur esprit, tandis qu'ils parvenaient au-delà d'eux-mêmes et de toutes les images $»^{18}$. Ce mystique lui ouvre le chemin vers le monde archétypal, car il y découvre que l'art n'est plus suspendu dans le vide : Ruysbroeck «nous a donné des racines ${ }^{19}$.

5 Les châteaux des pièces maeterlinckiennes, la fontaine dans le parc, l'anneau ${ }^{20}$, le cor sont des références claires, j’ai envie de dire stéréotypées, au Moyen Âge: le décor moyenâgeux des œuvres de Maeterlinck est une caractéristique connue. Cependant, ce Moyen Âge aperçu parfois comme permanence d'une vision romantique ou à la mode, 
parfois comme vision réactionnaire, tyrannique et sanguinaire du monde gothique, est - me paraît-il - surtout fondé sur l'idée de pouvoir puiser ainsi aux racines de la vie. Le cas d'une des pièces les moins connues au grand public, Joyzelle ${ }^{21}$, «charmant conte d'amour d'une clarté et netteté absolues", me paraît être un des plus significatifs ; cette pièce est liée au Moyen Âge ${ }^{22}$ par un rapport direct, stéréotypé suivant la mode de l'époque; par un rapport indirect, stylistique; et par un rapport profond, caché, expression en même temps du monde du Moyen Âge ${ }^{23}$ et de la Belgité moderne.

6 La critique a souvent présenté cette œuvre comme un pastiche, trop directement inspiré de La tempête de Shakespeare. Pour l'histoire, la pièce met en scène Merlin et Arielle, une femme fée invisible aux autres personnages, qui est le " génie » de Merlin. Ils habitent l'île du magicien et attendent le fils de Merlin, Lancéor, et la rencontre de celui-ci avec sa fiancée, Joyzelle. Le choix du personnage fait penser à un choix à la mode $^{24}$ : Merlin est un héros symboliste, consacré par le succès du tableau de BurneJones, «Merlin l'enchanteur » (1874) ${ }^{25}$, par l'exposition de 1878, et aussi par le poème de Jean Lorrain, «La forêt bleue » $(1883)^{26}$. Si la pièce est donc parfaitement inscrite dans son époque, la construction du texte paraît reprendre les mécanismes indirectement inspirés de Chrétien de Troyes ${ }^{27}$ :

Le retard dans la reconnaissance: Lancéor ne sait pas que Merlin est son père, que Joyzelle est sa promesse ; elle ne sait rien de lui, elle ne sait pas situer Merlin; les deux, ils ne savent même pas où ils se trouvent.

L'incertitude sur la nature des choses. Le décor est défini, mais seulement par des indications générales (une île, un château, un jardin sauvage) et avec parfois des renseignements, textuels et non didascaliques («c'est un brouillard épais qui nous a égarés ", p. 26).

Des îlots d'enchantement. Tout paraît définir un îlot d'enchantement. S'il n'y a pas d'objets qui bougent (comme dans le Perceval), il y a l'espace qui peut paraître truqué : «Elle regarde autour d'elle, stupéfaite; car dès l'entrée de Lancéor, sans qu'ils y aient pris garde, le morne jardin, s'est peu à peu magiquement transfiguré » (p. 55).

Une poétique de la merveille se développe et elle met en œuvre un jeu sur l'hésitation entre le naturel et le surnaturel : bien que Lancéor soit en train de mourir, après avoir promis à Merlin d'être à lui, Joyzelle voit son fiancé reprendre son souffle : "Que m'est-il arrivé ?... Je reviens, je reviens... j'étais couché là-bas, là-bas, je ne sais où, devant les grandes portes qu'on essayait d'ouvrir... J'étais enseveli et le froid me gagnait... » (p. 137).

7 Toutefois, c'est le sujet de la pièce qui mérite le plus d'intérêt, car il paraît résumer en soi une des problématiques qui marquent, en Belgique, la littérature du XIX ${ }^{\mathrm{e}}$ et du $\mathrm{XX}^{\mathrm{e}}$ siècle : le rapport au merveilleux ou la récupération de cet imaginaire qui choisit d'aller au-delà du réel pour dire le profond de l'âme. À la lecture de Joyzelle, on a l'impression que le choix de la mise en scène du merveilleux reproduise les mêmes thèmes que La seconde continuation du Perceval, avec la quête du Savoir absolu, la « Male Pucelle ", les épreuves à surmonter et le jeu qui appartient à une fée des eaux. Le merveilleux aide à jouer avec la simulation, et la possibilité de faire en sorte que les choses paraissent différentes de ce qu'elles sont.

8 Paul Valéry avait à dire, lors de l'inauguration du buste à Emile Verhaeren : «je me souviens toujours, Messieurs, de l'impression que j'ai éprouvée, il y a bien des années, quand j'ai lu l'étonnante préface que Maurice Maeterlinck a placée en tête de sa traduction de Ruysbroeck. Ce petit ouvrage me semblait contenir l'essence de toute une culture mystérieuse dont nous n'avions jusqu'alors quelque idée que par les célèbres peintures des maîtres de Bruges et de Gand $»^{28}$. Cette culture mystérieuse, Maeterlinck 
veut l'affirmer à travers le personnage fascinant et inquiétant de Merlin, à travers la nature double, bonne et mauvaise, de l'enchanteur. C'est un homme isolé, incompris, que les autres fuient puisqu'"ils sont persuadés que [1]a vertu secrète, à laquelle obéissent les plantes et les astres, l'eau, la pierre et le feu, [...] est cachée dans des philtres, des paroles maudites, des herbes infernales, des signes redoutables ${ }^{29}$; Merlin, lui, se sent, quoique magicien, victime de puissances occultes, de présences indéfinies qui pèsent sur la pensée. Il se sait être «menacé » par Viviane, celle que le mythe (et, au XIX ${ }^{\mathrm{e}}$ la tradition anglaise, avec Alfred Tennyson) veut à ses côtés, amoureuse et dangereuse, car elle va lui arracher son savoir magique ${ }^{30}$. Ce Merlin " humain » joue le rôle du père. Ainsi, semble-t-il être lié plus à la tradition allemande qu'à la tradition arthurienne de Robert de Boron ${ }^{31}$ : c'est seulement, en effet, dans le Merlin und Seifrid de Ardemont d'Albrecht von Scharfenberg que nous retrouvons parmi les changements importants apportés au personnage - celui d'un Merlin père, et ce sont les traductions de celui-ci qui voient le jour en Hollande et qui entrent dans l'imaginaire flamand. Bien qu'Apollinaire aussi eût écrit deux poèmes ${ }^{32}$ où l'idée de Merlin est présente, ce n'est que Maeterlinck qui s'attache aux problématiques du rapport père-fils ; un père qui veille sur le sort de son fils, Lancéor, qui doit rencontrer Joyzelle, sa fiancée.

9 Merlin vit dans l'angoisse d'être reconnu par son fils, qu'il ne voit pas depuis très longtemps, parce que "l'épreuve prescrite exige que nous vivions étrangers l'un à l'autre» (p.7). L'amour d'un père - dont le fils doit ignorer l'existence - ne doit pas fausser l'épreuve à surmonter ${ }^{33}$ : si Lancéor ne trouve pas l'amour "merveilleux", " ingénu », «clairvoyant », « héroïque », " plus doux qu'une fleur », "l'amour qui ne trompe pas", "que rien ne déconcerte", qui conduit à un avenir "mystérieux, invisible à tout autre ", il meurt. Cet amour est d'apparence " courtois ", idéalisé ; la femme « aimée » est une vierge qui doit tout comprendre et tout sacrifier à cela. Ainsi, comme la tradition le souhaite, cette vierge est la représentation de l'âme parfaite dans laquelle Dieu devient fécond ; Joyzelle est la terre qui regarde vers le ciel, une terre qui devient terre de lumière ${ }^{34}$; elle est aussi une femme qui écrase le serpent ${ }^{35}$; qui montre du courage et qui défit l'homme et le sort. Sa présence se croise avec celle d'Arielle, personnification de la «force intérieure » de Merlin ; c'est Merlin-femme ; ils sont une seule personne. On peut rappeler à ce sujet un mystique, l'allemand Maître Eckhart (maître de Ruysbroeck, par ailleurs), qui disait que l'homme devait garder sa virginité, et que pour ne pas renoncer aux fruits, il fallait qu'il devienne femme? Si je n'ai pas retrouvé encore d'affirmations précises sur les connaissances que Maeterlinck avait de ce mystique, je peux sans doute, aujourd'hui, déceler dans la construction du personnage de la pièce, dans ce personnage ambigu, un mécanisme d'introjection qui conduit au-delà du monde poétique, vers les «régions » que Maeterlinck lui-même définissait comme étant " plus profondes et plus intéressantes que celles de la raison et de l'intelligence $~^{36}$. En effet, dans la dialectique des rapports à l'Autre, l'interjection d'un trait de l'autre (en ce cas la féminité), constitue un signifiant, qui, en même temps qu'il fait surgir le sujet (Merlin agit à travers elle), le réduit à n'être que ce signifiant. Ainsi, la séparation voulue d'Arielle qui se vérifie dans la pièce, ( "C'est pour m'ouvrir les yeux et non pour m'égarer que je t'ai tirée de ton ombre impuissante », p. 102) n'est qu'illusoire, car elle ne peut pas, normalement, être vue par les autres. Cette séparation devient l'expression de la perte que le sujet a subie. Il la concrétise, en lui donnant la forme des objets primaires de sa pulsion, une première femme fragile, mais toute puissante. De plus, symboliquement, n'est-ce pas cela une forme d'« humanisation »du 
mysticisme ? Si celui-ci est un ensemble de croyances se donnant pour objet une union intime de l'homme et du principe de l'être, du divin, l'introjection de la femme « divine » ne serait-elle pas aussi une façon d'englober la mystique flamande?

Arielle-Merlin, pour mettre à l'épreuve l'amour des deux jeunes, se montre à Lancéor, «à demi nue et son pied recourbé comme une fleur prudente, tâte l'eau qui sourit en le cerclant de perles» (p. 71). Lancéor prend dans ses bras cette femme manifestement très sexuée et l'embrasse ardemment : " je la vois qui se penche, je la vois qui consent " (p.72). Cet amour incestuel ${ }^{37} \mathrm{du}$ père est doublé du désir de Merlin-Arielle pour Joyzelle, amour avoué et niés ${ }^{38}$ : si elle ne se donne pas à lui, Lancéor sera tué, mais Joyzelle préfère la tentative de tuer elle-même Merlin, qui sera sauvé par Arielle; Merlin avoue, alors, que tout revenait à sa tentative de provoquer l'honnêteté de Joyzelle pour être sûr du bonheur futur de son fils. La volonté de Merlin de viser l'absolu pour son fils et sa fiancée, et cela à travers la provocation sexuelle à l'un et à l'autre, instaure un mécanisme dans lequel le père vise le bien de ceux qu'il aime à travers la souffrance. Ce procédé initiatique, qui commence quand Joyzelle et Lancéor découvrent l'amour - un amour idyllique auquel Merlin met obstacle à travers sa propre personne - se croise avec les problèmes d'un Merlin qui doit se soumettre au destin, "aux pouvoirs inconnus ", et " interroger les faits comme les autres hommes; attendre leur réponse, et tenter de les vaincre s'ils veulent le malheur de ceux que nous aimons " (p.13). Pour ce faire, il joue en même temps le rôle de père méchant (qui n'aime pas son enfant et pense seulement à satisfaire son propre désir le plus instinctuel) et de père idéal (qui se préoccupe du bonheur de son fils et veut " contrôler» que la femme choisie soit véritablement la «femme idéale " pour lui). Dans les deux cas, c'est une histoire perverse ${ }^{39}$, qui, dans son dénouement heureux ${ }^{40}$, est présentée seulement du point de vue irénique, car «le reste n'appartient pas encore aux hommes » (p. 177). Une histoire perverse et incestueuse dont le monde féerique moyenâgeux couvre la complexité profonde. Cette initiation sans connaissance (car la raison pour laquelle il fallait faire tout cela reste inconnue), ce bonheur sans connaissance (car Lancéor ne connaît pas l'épreuve que sa fiancée a dû subir) disent le fictif, non pas (ou non seulement) de l'univers dans lequel ils ont pris forme, mais surtout de la pensée qui les a produits ; et cela à travers le personnage d'Arielle ( ma fille invisible, la bonne fée de l'île», p. 175). Cette femme invisible, en même temps compagne de vie et fille, ce personnage qui réalise tous les désirs les plus secrets, n'estce pas la vision de la mère, du personnage féminin avec lequel espérer une union totale, originaire ${ }^{41}$ La psychologie des personnages est donc faussée par cet aspect ambigu bien en consonance avec l'ambiguïté de Merlin. En effet, le père réel est celui qui permet à l'enfant d'avoir accès au désir sexuel, celui qui permet au garçon d'avoir une position virile. Pour faire cela, toutefois, il convient que le père réel possède l'atout maître, le pénis réel ${ }^{42}:$ l'interdit ne peut faire passer le sujet à une position sexuée qu'à la condition que la mère, interdite pour lui, ne soit interdite que parce que le père la possède. Or, il n'y a pas de mère dans le texte ${ }^{43}$. L'espoir que le fils puisse devenir « ce que nous savons bien que je ne saurais être» (p.11), reste tel: nous ne saurons pas comment l'histoire se termine.

11 Si je vois bien toute la valeur du «sadisme » que d'autres ont mis en évidence, je me permets toutefois de nuancer. La définition de Maeterlinck (le sadisme est une « exacerbation de l'intérêt et de l'amour que le poète porte à ce qui l'environne »4), partagée par les critiques, me paraît édulcorée par rapport à l'amour fusionnel et anéantissant mis en scène. La comparaison proposée par Pouillard entre Maeterlinck et 
Thomas De Quincey, que je ne veux certes pas nier ici, me paraît dissimuler cet aspect profond de retour aux origines qui va de pair avec l'expression d'un malaise psychologique profond. Le renvoi au monde anglo-saxon comme source d'inspiration continue de brouiller les pistes pour la compréhension de l'auteur et de la littérature belge et ne permet pas de situer le problème qui est dans la reprise de ce qu'a caractérisé le Moyen Âge: le passage du monde païen au monde catholique; le fusionnement de ces deux aspects dans un catholicisme qui cherche à englober tout et le définit. Si le gothique se forge sur la "monstruosité » de l'imaginaire qui en découle (et donne une place importante au diable et à la peur, l'angoisse), si le XIX ${ }^{\mathrm{e}}$ siècle français cherche à le "bloquer » dans des images figées, les belges vivent ce gothique comme étant le résultat d'un passé qui leur appartient. Ce passé a perdu sa fonction de connotation et de repère temporel pour représenter un temps qui est toujours présent, le choix entre une vie païenne et une vie catholique, une vie sexuelle et une vie chaste, une vie perverse et une vie «normale » étant toujours le problème à résoudre.

La mystique flamande ${ }^{45}$, ces ardeurs qui « semblent à présent de fiévreuses ténèbres ", assume toute sa valeur. Interroger les textes du Moyen Âge, s'inspirer d'eux, y puiser style et forme, fasciné par la mystique qu'on y retrouve, forge l'inconscient esthétique (au sens étymologique, de sentiment) de l'écriture, un inconscient qui cherche à travers le merveilleux à laisser dans l'ambiguïté des sous-entendus les mécanismes profonds de l'âme. Cette mystique ne peut que paraître, dès lors, que ténébreuse, quoique toujours enfiévrée. Nordau trop dur dans ses affirmations, n'a pas tous les torts quand, en prenant en compte dans son œuvre ${ }^{46}$ l'auteur belge, voit l'écrivain comme un dégénéré et ses textes comme une parodie du mysticisme («son mysticisme devenu absolument enfantin et idiotement incohérent»). Il insiste toutefois sur l'importance anglosaxonne dans l'œuvre de Maeterlinck, qui ne serait qu'une anthologie shakespearienne. Si sous certains aspects matériels, on partage cette affirmation, il faut cependant regarder plus profondément et se tourner vers $L a$ mystique flamande ${ }^{47}$. Elle se caractériserait, comme Maeterlinck le dit, par la bonne foi, la sincérité et la familiarité dans l'abstrait. Joyzelle n'en est-elle pas l'exemple le plus pur? «Bonne foi » de Merlin, sincérité des personnages; reste le problème du sens à donner à " familiarité »; peutêtre «abandon », se laisser aller ? La familiarité des sentiments qu'on met en scène ne peut être acceptée que dans l'abstrait. À Maeterlinck de souligner que l'activité spirituelle - qui, dit-il, précède la peinture flamande pendant plus de deux siècles - a laissé ses marques dans le peuple des Pays-Bas et lui a offert une vision de morale ascétique. Les Flamands, depuis lors, depuis ces mystiques, savent évoluer vers la pensée abstraite. Le mysticisme est ainsi, pour Maeterlinck, sublimation-dans la pensée, dans le merveilleux, dans le rêve - de ses propres désirs (même pervers) : à cela toutefois Maeterlinck ne voudra plus attacher d'importance après que Freud eût démontré que trop de choses ne sont pas liées à la raison. Ses rêves « fidèles ", un vol plané ${ }^{48}$, n'auront plus d'importance. Est-ce trop y voir une affirmation implicite à ce que je viens de dire? C'est que le vol est sublimation (même de l'acte sexuel), métaphore de ce qu'on n'est pas capable de réaliser sur terre, simulation du mythe d'Icare pour fuir soi-même, pour une élévation vers le ciel qui ne prendra jamais son élan définitif.

13 Ne voulant certes pas nier ici l'existence d'une influence anglo-saxonne, ou allemande ${ }^{49}$ que l'œuvre de Maeterlinck dévoile, il me plaît toutefois de mettre en évidence l'importance qu'a pour l'écrivain belge le mouvement européen du Moyen Âge, ce monde polyglotte qui est à la recherche de sa propre identité, qui trouve souvent 
l'expression de soi dans l'humour, qui réagit à l'église à travers le merveilleux, qui pense le destin de l'homme transfiguré par la souffrance. C'est à cette époque que prennent forme des mutations importantes. La mystique flamande, comme Maeterlinck la percevait, dit la recherche de l'absolu de la part de l'homme et la difficulté de celui-ci à l'atteindre, vivant toujours dans l'entre-deux, comme au Moyen Âge : pas encore affranchi du paganisme, avec ses obsessions sexuelles, son agressivité, son sens de la vengeance tenant lieu de justice, il partage cependant de plus en plus la force qui émane de la christianisation. C'est la transfiguration de l'homme par la sainteté, catholique ou laïque quelle qu'elle soit.

Ainsi la lecture de ces textes faite seulement avec une clé préraphaélite, brouille les pistes et ne permet pas la compréhension des problématiques profondes de l'œuvre.

L'ambiguïté textuelle et interprétative - fondée sur les pouvoirs d'une tradition moyenâgeuse que l'on découvre dans les textes de Maeterlinck - se retrouve aussi chez un autre grand écrivain belge de la fin du XIX ${ }^{e}$ siècle, Emile Verhaeren. Son monde du merveilleux témoigne à la fois d'un refus et d'une fascination incontournable pour ses racines flamandes moyenâgeuses ; ce dédoublement est fortement présent surtout dans ses premiers textes.

Conte gras $^{50}$, par exemple, raconte une fresque - dans une belle maison anversoise - qui se « heurte » à un tableau gothique et en meurt. Le protagoniste Ernest Vinckx, qui vit cloîtré dans cette maison, n'aime que «l'art gras, l'art qui procure la paix d'une bonne digestion» (pp. 6-7), « la sensualité des vieux maîtres, les saturnales païennes » (p. 8). Depuis 10 ans, il ne quitte plus sa chambre, quand, un jour, il reçoit en héritage un tableau gothique du $\mathrm{XV}^{\mathrm{e}}$ siècle, emblème de la mort et de la maladie : « un long Christ osseux, bistre, le front échiqueté d'épines, les yeux pourris, les mains béantes » (p. 10). Mais Vinckx, y découvre " une beauté malade, insoupçonnée » : le charme qui émane de l'œuvre le hante, la scène de torture le captive, et Vinckx ne s'aperçoit pas du trouble que le tableau suscite parmi les hôtes immobiles de la chambre, parmi les habitants de sa fresque. Ce malaise général, qui dit que les choses ont des sentiments humains, annonce leur mort. Ils se liquéfient : d'abord une goutte, puis une averse et encore une coulée épaisse, de gras. La salle entre en décomposition. Vinckx s'enfuit, crie de terreur; il croie à une hallucination: les chairs en fleurs de son Olympe paraissent mortes ${ }^{51}$. La résurrection attendue et espérée après ce geste libératoire ne se fait pas : le charme de la mort et de la souffrance l'avait conquis ; la religion lui avait fait découvrir «le charme hantant » d'un Christ souffrant sur la croix.

Dans ce conte, Verhaeren paraît suivre les mécanismes rhétoriques du Moyen Âge, quand tout énoncé poétique avait quatre sens : littéral, allégorique, tropologique et anagogique. Le sens littéral nous conduit vers le monde fantastique où les fresques peuvent être effacées par des forces mystérieuses ; l'allégorique souligne l'aspect de la joie de vivre tuée par la force de la religion; le tropologique prend forme dans cette chambre qui représente le monde de cet homme et sa façon d'être ; et l'anagogique, qui permet de représenter de façon plus abstraite un objet de pensée, trouve sa focalisation dans le problème de la maladie, implicite dans la figure du Christ maigre, de cette maladie qui fascine, mais qui tue. Le texte lui-même devient, alors, la représentation de tout ce qui, aimé (l'art gras, les saturnales païennes), est détruit par ce qu'on n'aime pas ("Vinckx ne goûtait guère le génie du Moyen Âge», p.11) parce qu'il est inconsciemment en nous et est subi par tradition (« un jour il recueillit par héritage un tableau gothique » p. 10). Verhaeren dit subtilement l'ambiguïté du rapport au Moyen 
Âge, le charme et le refus qui en découlent, aussi en utilisant un mécanisme d'opposition à la tradition biblique des quatre sens: le littéral met en scène le fantastique et non l'historique, l'allégorique (relatif aux mystères du Christ) met en scène une religion qui tue ; le tropologique (l'appelle à la vertu), la contemplation des saturnales et l'anagogique (contemplations des choses cachées) le charme du physique.

Dans ce discours constamment double pour Verhaeren, le Moyen Âge est «barbare et grand $»^{52}$ : on ne l'aime peut-être pas, mais il conditionne ; on cherche à s'affranchir de lui, mais il garde le poids d'un saint qui repose dans une châsse énorme, comme s'il s'agissait d'un reliquaire ${ }^{53}$. Symbole d'un passé refoulé, il en a la force : il motive et paralyse ; il vivifie et meurtrit en même temps : à travers sa force, sa sauvagerie et sa culture. Cette vision est chère aussi à Georges Eekhoud, qui - par exemple dans Cycle patibulaire $^{54}$ et dans le conte Les crois processionnaires - met en scène encore une fois la grandeur et la barbarie du Moyen Âge, scénario du cycle inquiétant de l'homme, nouveau Christ dont la vie est un constant "patibulum», déterminé par des lois absurdes ; elle est chère aussi à Franz Hellens ou Michel de Ghelderode, pour lesquels la symbologie du Moyen Âge est utilisée comme De Heredia a pu le faire, le décor se fixe "dans un Moyen Âge stylisés ${ }^{55}$ ». Le Moyen Âge, aimé ou pas, est une source vitale, l'archétype profond de l'imaginaire d'un peuple.

Un Moyen Âge a-temporel

La seconde moitié du XXe siècle a pourtant vu s'atténuer certaines catactéristiques : les écrivains d'origine flamande écrivent de moins en moins en français et du Moyen Âge il ne reste que la seule valence négative, un refus, souvent vécu dans le cynisme. Thomas Owen en est un exemple important.

Cet écrivain a souvent fait appel à un imaginaire du Moyen Âge (à titre d'exemple, je cite : La belle vaincue et le troubadour ${ }^{56}$; La nuit au châtea ${ }^{57}$; Le page; Le châtelain ${ }^{58}$; Le tetrastome ${ }^{59}$ ) avec des rappels explicites ou implicites. Explicites si l'on considère que par exemple La nuit au château semble lancer un clin d'œil au Perceval, ou peut-être à Gauvain: un château et un monde chevaleresques, une dame et son mari; le chevalier qui entre à cheval dans le hall du château; les lois de la courtoisie et de l'hospitalité ; le châtelain qui reste hors de scène; l'infidélité de la dame; le rôle de la chasse; le chevalier au service des vouloirs de la dame. Même les thèmes choisis sont les mêmes : la tentation; la volonté de tromper le chevalier; la mort inéluctable en épilogue; le sens du devoir. Explicite est aussi le rappel au monde chevaleresque à travers le lexique qui lui est propre. La belle vaincue et le troubadour est l'histoire de deux hommes : l'un, chasseur, suit de l'œil un bateau du bord du fleuve. L'autre, un troubadour joue de la guitare sur le bateau; à côté de lui, une femme splendide, détendue et nue, se laisse bercer par le mouvement de l'eau. Pris par la situation édénique, le troubadour n'écoute que sa musique, alors que le chasseur ne voit qu'à travers son arme, et il tire : « la guitare éclata comme un ballon de verre. Le pauvre chanteur, de voir brisé ainsi son précieux instrument, arrêta là sa chanson et contempla, triste et surpris, ses paumes vides et son pouce qui cherchait des cordes à gratter $»^{60}$. L'instrument authentique "violon d'Ingres ${ }^{61}$, représentation symbolique du corps féminin et moyen pour l'atteindre à travers la sublimation - a disparu ; l'artiste ne peut alors que se tuer. Si même le symbolique ne lui est plus permis par la force virile que le chasseur dévoile, il ne peut qu'opérer un regressum ad uterum: ainsi, sans rien dire, il se jette dans l'eau et se noie face à l'indifférence de la belle dame qui descend du bateau et 
reprend sa vie normale avec celui que le texte laisse supposer être son mari. Le monde moyenâgeux n'offre que des rêves hors du temps.

21 Les rappels implicites sont très fréquents aussi; ce sont toujours les mêmes que Maeterlinck avait déjà présentés : le rôle du père surnaturel, les objets magiques, le cor, l'anneau, « le fier baiser » ou la main coupée. Dans un cas ou dans l'autre, en tout cas, la «mystique » moyenâgeuse est devenue plus cynique. La femme, aérienne, belle, nue, la femme dont la pureté serait la valeur de la vie de l'homme, existe seulement dans la pensée de l'homme. C'est un idéal, comme celui de la musique, ou de l'art, qui tue, qui n'a pas de place en ce monde : le penser c'est donner forme à sa rage et à ses pensées les plus profondes et cachées. L'homme s'ouvre ainsi au monde de ses pulsions, qu'il entoure d'un halo magique, pour ne pas y voir clair. Le châtelain, qui a " une netteté » profonde, inquiète ${ }^{62}$. C'est un chanteur horrible qui joue avec les sortilèges, qui peut induire la paix au toucher, un "soulagement calme ». C'est encore Merlin, non pas dans sa complexité refoulée, mais accepté dans sa perversité : il peut changer les choses et s'introduire dans le monde d'autrui à son gré. Il regarde au monde « les yeux rieurs", car "les temps sont diablement incléments aux troubadours de mon espèce ». Le monde du Moyen Âge continue de vivre dans les mœurs, dans le collectif, mais il est devenu surtout le moyen pour dépasser un manque, un quelque chose qui n'est plus : "Les bonnes gens m'appellent le Châtelain. Je n'ai pas de château ». Le châtelain n'a plus de châteaux, emblématique représentation d'un quelque chose qui est, de par sa collocation, un endroit isolé, et une forteresse de puissance, expression d'un pouvoir inatteignable et mystérieux. Expression aussi du transcendant. Ce sont les châteaux de l'âme des mystiques, les demeures successives dans la voie de la sanctification. Les châteaux mystérieux et lugubres, présents chez Maeterlinck, ne sont plus. Expression d'abord de la tour d'ivoire dans laquelle un poète pouvait se renfermer avec toutes ses magies, illusion aussi d'un passé avec ses défauts mais existant, force provocatrice pour dénicher le mal, le Moyen Âge et avec lui ses symboles, paraissent devenir de plus en plus, dans ce monde francophone qui s'éloigne de ses "frères" flamands, l'emblématique expression d'un rêve qui n'existe plus, le rêve d'une identité complexe dont le Moyen Âge avait été porteur.

\section{NOTES}

1.Cf. à ce sujet les actes du colloque qui a eu lieu en juin 2000 à l'Université de Versailles-Saint-Quentin-en-Yvelines, publiés par les soins de Laura Kendrick, Francis Mora et Martine Reid, Le Moyen Âge au miroir du XIX ${ }^{e}$ siècle (1850-1900), Paris, L'Harmattan, 2003.

2.Cf. à ce sujet G. Charlier, J. Hanse, « La culture française à la cour des ducs » in G. Charlier, J. Hanse, Histoire des lettres françaises de Belgique, Bruxelles, La Renaissance du Livre, 1958, p. 83.

3.Charles De Coster commence à rédiger La Légende en 1858 et la termine en 1864. Il attend pendant trois années les dessins de Félicien Rops avant de pouvoir publier. Le volume paraît avec la moitié des illustrations prévues en 1867. Ce texte est considéré 
aujourd'hui comme un chef-d'œuvre des lettres françaises : « si Romain Rolland a proclamé sa valeur d'art universelle, si Georges Duhamel l'a mise au rang des grandes épopées, à côté de l'lliade, [...] si la littérature néerlandaise, par ses traductions et ses imitations, à véritablement adopté cette œuvre française », en Belgique et en France l'intérêt aura un parcours plus lent (Cf. À ce sujet, G. Charlier, J. Hanse, Histoire illustrée des lettres françaises de Belgique, cit., p. 305 et svv.).

4.Dans un article du « Soir » du 8 janvier 1977 il est écrit : «Ce Thyl n'est pas seulement espiègle, c'est un homme du Moyen Âge, fils du peuple, solidement ancré dans son époque et qui lutte pour la liberté ».

5.Cf. J. Gousseau, Charles De Coster à l'écran, in La Littérature belge de langue française, "Itinéraires et contacts de culture », Paris, L'Harmattan, 1995, pp. 163-173.

6.Il s'agit de l'épisode dans lequel Ulenspiegel s'approche du bûcher où est Claes; le sergent lui demande : « Sorcier, cherches-tu des mains de gloire?»(p. 127). Nombreuses sont les références aux sorciers dans le texte : l'histoire du poissonnier, par exemple : « Je ne veux rien dire. J'ai parlé trop : vous me brûlerez. Je ne suis point sorcier, pourquoi me replacer près du feu?» (p. 312). Katheline aussi est définie la « bonne sorcière » (p. 10) ou la sorcière mignonne (p. 340) et Joos est un sorcier (p. 341, p. 355) (Ch. De Coster, La légende de Thyl Ulenspiegel, Bruxelles, Éditions de la Toison d'or, 1942, Collection « La porte ouverte »).

7.C'est ainsi que nous l'apercevons lors de la première entrée en scène de Philippe enfant, en train de brûler une guenon : « la bestiole avait tant souffert en mourant dans ce feu que son petit corps semblait être [...] un fragment de racine rugueuse et tordue, et dans sa bouche ouverte comme pour crier la mort, se voyait de l'écume sanglante, et de l'eau de larmes mouillait sa face. [...] Philippe, tout noir vêtu et suçant un citron » attendait dans un coin, à l'ombre, Ch. De Coster, op. cit. pp. 30-31.

8.Cf. D. Poirion, Le merveilleux dans la littérature française du Moyen Âge, Paris, PUF, 1995, p. 27.

9.« Fleurs de force et de santé féminines, nues et point rougissantes, fières de leur beauté, n'ayant pour tout manteau que leurs chevelures [...] Ce sont elles qui errent le soir dans les parcs et jardins [...] Sitôt que passent devant elles un jeune gars et une fillette, elles essaient de tuer la fillette, mais, ne le pouvant, soufflent à la mignonne, encore résistante, désirs d'amour afin qu'elle se livre à l'amant ; car alors la fille-fleur a la moitié des baisers ", Ch. De Coster, op. cit., p. 150.

10.Comme dans l'épisode du chapitre 42 où l'empereur arrive à Audenaerde, en Flandre, et n'est pas reçu comme il se doit à cause de Ulenspiegel. Le héros est condamné à mort, mais le peuple rassemblé demande sa grâce. Sur cela l'empereur décide : "Si ce vaurien me demande quelque chose que je ne puisse faire, il aura la vie sauve ». Et Ulenspiegel de demander : « Majesté, je demande qu'avant que je sois pendu, vous veniez baiser la bouche par laquelle je ne parle pas flamand ». L'empereur, riant ainsi que tout son peuple, répondit : « je ne puis faire ce que tu demandes, et tu ne seras pas pendu, Ulenspiegel ». Ch. De Coster, op. cit.

11.M. Maeterlinck, « Introduction » à L'ornement des Noces spirituelles, de Ruysbroeck l'Admirable, traduction de M. Maeterlinck, Paris, Paul Lacomblez - Nilson, 1891. Le texte a été repris dans l'Introduction à une psychologie des songes, Bruxelles, Labor, 1985, pp. 63-78.

12.« Il a supposé la plupart de ses prédécesseurs inconnus », Ibidem, p. 66.

13.Ibidem, p. 69.

14.Ibidem, p. 68. 
15.Ibidem, p. 64.

16.Ibidem, p. 69.

17.La découverte de Ruysbroeck est déterminante dans l'évolution littéraire de $M$. Maeterlinck. On a longtemps cru que c'était Villiers de l'Isle Adam qui avait introduit Maeterlinck dans la période mystique, aussi parce que l'écrivain lui-même l'avait déclaré dans la (désormais) fameuse enquête littéraire de Jules Hurlet (1891) : « tout ce que j'ai fait, c'est à Villiers que je le dois ». Aujourd'hui, les correspondances ont élargi les connaissances. De plus, une dédicace à Huysmans dit : « au Maître profondément admiré qui m'a mis sur la voie de Ruysbroeck » (Paris, Bibliothèque J. Doucet, volume exposé à l'exposition Maeterlinck et repris dans le catalogue de l'exposition même édité par les soins de J. Warmoes, Bruxelles, Bibliothèque Albert $1^{\mathrm{er}}$ en 1962).

18.M. Maeterlinck, « Préface » à L'ornement des Noces spirituelles, de Ruysbroeck l'admirable, "La revue générale », octobre-novembre 1889, p. 664. Cet extrait n'est pas repris dans l'édition de l'œuvre de 1891.

19.Ibidem, p. 668.

20.Cf. notamment C. Lutaud, «Le mythe maeterlinckien de l'anneau d'or englouti », Annales de la Fondation Maeterlinck, XXV, 1979, pp. 63-67.

21.M. Beaubourg, « Critique des théâtres. Joyzelle », in La Plume du 15 juin 1903.

22.Cf. A ce sujet aussi l'article de A. Rykner, "Le drame symboliste », La fabrique du Moyen Âge. La réception de la civilisation médiévale dans la littérature française du XIX ${ }^{e}$ siècle, éd. S. Bernard-Griffiths, P. Glaudes, B. Vibert, Paris, Champion, 2003.

23.Parmi les nombreux essais consacrés à ce sujet cf. C. J. Lambert, The empty cross : medieval hopes, modern futility in the theater of Maurice Maeterlinck, Paul Claudel, August Strinberg and Georg Kaiser, New York, Garland Publ., 1990.

24. La pièce date de 1903, mais le symbolisme belge a une vie plus «longue » que le symbolisme français. D'ailleurs, c'est en 1904 que Charles Van Lerberghe publie le texte considéré comme le chef-d'œuvre du mouvement, La Chanson d'Ève.

25. À relever que Viviane est citée, dans le texte, comme celle qui attend Merlin s'il ne sait pas saisir l'occasion d'un amour différent comme celui pour Joyzelle, voir aussi note 24 .

26.La forêt bleue est par ailleurs citée dans la pièce : le protagoniste est au bout de l'île, près de la forêt bleue qui ferme l'horizon (p. 44).

27.Je reproduis la structure proposée, pour l'analyse de Chrétien de Troyes, par Daniel Poirion, op. cit.

28. Manuscrit autographe présenté à l'exposition pour le Centenaire de Maurice Maeterlinck (collection Jean Goffin) et repris dans le catalogue Maurice Maeterlinck de Jean Warmoes, imprimé à Bruxelles, Bibliothèque Albert $1^{\mathrm{er}}$ en 1962, p. 54.

29.M. Maeterlinck, Joyzelle, pièce en cinq actes (représentée pour la première fois le 20 mai 1903), Paris, Librairie Charpentier et Fasquelle, 1914, pp. 4-5. Première édition chez Fasquelle en 1903.

30. “ C'est le nom de la fée, qui dans Brocéliande, où ton destin te mène, attend que tu paraisses pour briser ta vieillesse... Oh ! Maître ! je la vois !... Prends garde, elle s'approche et va gagner ton cœur !... Dès que cet amour-ci, si pur, si salutaire, aura perdu ses droits, le sien rampe hors de l'ombre... Maître ! je t'en supplie !... Mes yeux comptent ses ruses, elle t'enlace de ses bras qui parodient l'amour, elle te prend la puissance, la raison, la sagesse ; elle t'arrache enfin le secret de ta force ; et comme un vieillard ivre, tu tombes sur le sol... Alors elle te dépouille, te raille, se redresse et referme sur nous la caverne mortelle qui ne s'ouvrira plus... » (p. 103). 
31. Je partage en cela l'opinion de P. Gorceix (Maurice Maeterlinck, « La mystique médiévale et le symbole ", in La licorne, VI, 1982, pp. 51-64), parfois discutée puisqu'elle ne donne pas assez d'importance à la tradition arthurienne.

32. Il s'agit de « Merlin et la vieille femme » (Alcools, 1900) et de L'enchanteur pourrissant de 1904-1909; le tombeau de Merlin devint le point de convergence des mystères de la vie, de l'amour et de la mort.

33. "Le destin de ton fils est inscrit tout entier dans un cercle d'amour » (p. 8).

34. "Sa douce chevelure s'épanche comme un flot de lumière immobile ; et la lune ne sait plus à qui appartient l'or qui se mêle à l'azur où glissent ses rayons » (p. 98).

35.Lancéor : « Joyzelle ! Je suis blessé... Un serpent m'a mordu...

Joyzelle: «Ce n'est pas un serpent... C'est une bête affreuse !... Elle se dresse contre toi !... Je l'écrase sous le pied... Elle bave... Elle est morte ", p. 63.

36.Cf. Les pages écrites pour « Le soir » du 19 août 1932 par Eugène Baie, pour commémorer le soixante-dixième anniversaire du poète, reprises aussi dans Maurice Maeterlinck, catalogue édité par J. Warmoes, op. cit., pp. 123-127.

37.J'utilise ici la définition de Paul Claude Racamier, (L'inceste et l'incestuel, Paris, éditions du Collège) qui prend en considération aussi le climat, qui, dans la vie familiale individuelle et collective, crée l'empreinte de l'inceste.

38.« Non, Claude, à mon âge on ne se contente plus d'illusions [...]. L'heure est passée pour moi du mensonge héroïque. Je veux ce qu'il aura. Peu m'importe ton sourire que je sais impossible, c'est toi-même que je veux; je te veux tout entière, ne fût-ce qu'un moment ; mais j'aurai ce moment ; celui-ci me le donne » (p. 133).

39.Quoique pour des raisons différentes, je me trouve assez en accord avec la pensée de Camille Hanlet (Les écrivains belges contemporains, Liège, Dessein, t.1, 1946, pp. 219-242 et « Maurice Maeterlinck, le danger d'une œuvre littéraire ", La pensée catholique, 1942, p. 32) qui jugeait l'œuvre de Maeterlinck « une œuvre essentiellement malsaine ». Cf aussi R. Campagnoli, «Les Serres froides de Maeterlinck », in Lettres ou ne pas lettres, Mélanges de Littérature française de Belgique offerts à Roland Beyen, par J. Herman, L. Tack et K. Geldof, Leuven, Presses Universitaires de Louvain, 2001, pp. 47-55.

40.Claude : «Non, non, non, non, mon père, vous ne partirez pas !... Quand on est à trois qui s'aiment, à subir un malheur, le malheur se transforme en un fardeau d'amour que l'on porte avec joie... (p. 176).

41.L'union totale est une présence forte dans les textes de Maeterlinck. Je renvoie par exemple à Aglavaine et Sélysette : « lorsque je t'embrasse à mon tour, il me semble que c'est moi-même que j'embrasse [...] je ne suis réelle que lorsque tu es là [...] Je me cherche hors de moi et c'est en toi que je me trouve, je te cherche hors de moi et c'est en moi que je te trouve), in M. Maeterlinck, Aglavaine et Sélysette, Acte II, sc. I.

42.Cf. R. Chemama et B. Vandermersch, Dictionnaire de la psychanalyse, Paris, Larousse, 1998.

43.Problème par ailleurs récurrent. Cf. à ce sujet, Ana Gonzalez Salvador, La pièce qui fait défaut. Lecture d'onirologie de M. Maeterlinck, in « Textyles », 19, 1993, pp. 59-71.

44. Cf. l'essai de R. Pouillart, Maurice Maeterlinck. Subconscient et 'sadisme', in « Les lettres romanes », XXVII, 1973, pp. 37-61.

45.Problème qui a suscité de nombreuses discussions, notamment sur la réception de Maeterlinck aux Pays Bas. Cf. à ce sujet l'imposante bibliographie maeterlinckienne de A. Rickner, Maurice Maeterlinck, Paris-Roma, Memini, 1998.

46.M. Nordau, La dégénérescence, Paris, Alcan, 1895, pp. 442-463. 
47.M. Maeterlinck, « La mystique flamande », Revue encyclopédique, 24 juillet 1897, pp. 626-627.

48.Cf. « Des rêves ", une enquête que Franz Hellens et Henri Michaux, alors directeurs du Disque vert avaient effectué en 1925 et la réponse que Maeterlinck donna à la question : « avez-vous des rêves fidèles ? » Le Disque vert, vol. II, Bruxelles, Editions Jacques Antoine, 1970.

49.L'influence, très présente, est surtout liée aux traductions de Shakespeare que l'auteur fait. Pour l'influence allemande voir P. Gorceix, Les affinités allemandes dans l'œuvre de Maurice Maeterlinck. Contribution à l'étude des relations du symbolisme français et du romantisme allemand, Paris, PUF, 1975.

50.E. Verhaeren, Contes de minuit, Bruxelles, Finck éditeur, 1884.

51.Ibidem, « Toute cette beauté en rouge était fondue, ses rayonnantes déesses évanouies. Il devina : arracha le tableau gothique et le jeta dehors. Ils agonisaient à cause de cela. La maigreur était contagieuse, les grasses avaient été vaincues » (p.19). 52.E. Verhaeren, « Les crucifères », Les moines, Paris, Alphonse Lemerre, 1886.

53.E. Verhaeren, Les moines, Paris, Alphonse Lemerre, 1886.

54.G. Eekhoud, Le Cycle patibulaire, Bruxelles, Kistemaeckers, 1892.

55.Cf. "L'Alérions », in Poèmes, 1887-1892, Paris, Mercure de France, repris aussi in $\mathrm{H}$. Morier, Dictionnaire de poétique et de rhétorique, Paris, PUF, 1989, « allégorie ».

56.Thomas Owen, Cuvres complètes, Bruxelles, Lefranca, 2000, volume III, p. 159.

57. Ibidem, p. 167.

58.Ibidem, volume I, p. 989.

59.Thomas Owen, Le Tetrastome, Bruxelles, Éditions Bernard Gilson, « Pré aux sources », 1990.

60.Thomas Owen, op. cit., p. 162.

61.Cf. La photo de Man Ray de 1924.

62.Th. Owen, op. cit., p. 992.

\section{AUTEUR}

\section{ANNA SONCINI}

Université de Bologne 